\title{
Promotores de crescimento na propagação de caroba
}

\author{
Patrícia Pinheiro Amaral ${ }^{1}$, Gerusa Pauli Kist Steffen ${ }^{2 *}$, Joseila Maldaner ${ }^{2}$, Evandro Luiz Missio², Cleber Witt Saldanha ${ }^{2}$ \\ ${ }^{1}$ Universidade Estadual do Rio Grande do Sul, Rua Sete de Setembro, 1040, CEP 96508-010, Cachoeira do Sul, RS, Brasil \\ ${ }^{2}$ Fundação Estadual de Pesquisa Agropecuária, BR 287, Acesso VCR 830, Km 4,5, CEP 97001-970, Santa Maria, RS, Brasil
}

*Autor correspondente:
gerusa-steffen@fepagro.rs.gov.br

Termos para indexação:

Jacaranda micrantha

Vermicompostagem

Trichoderma

Index terms:

Jacaranda micrantha

Vermicomposting

Trichoderma

Histórico do artigo:

Recebido em 02/02/2017

Aprovado em 09/06/2017

Publicado em 30/06/2017

doi: $10.4336 / 2017 . p f b .37 .90 .1402$
Resumo - O uso de insumos biológicos capazes de promover crescimento vegetal representa uma alternativa para antecipar a comercialização de mudas florestais em condições de viveiro. A caroba (Jacaranda micrantha) é uma espécie florestal nativa da região sul do Brasil, que apresenta diversos potenciais de uso. O objetivo do trabalho foi analisar a eficiência da utilização de vermicomposto e ou isolados fúngicos do gênero Trichoderma como promotores de crescimento de mudas de J. micrantha. Foram avaliados nove tratamentos contendo diferentes percentuais de vermicomposto $(0,25$ e $50 \%)$ no substrato padrão (solo peneirado e substrato comercial Carolina Soil ${ }^{\circledR}$ na proporção 1:1), na presença ou ausência de $T$. asperelloides e $T$. virens. Utilizou-se delineamento inteiramente casualizado com 18 repetições por tratamento. Aos 90 dias após a semeadura em tubetes, determinou-se: altura da parte aérea, número de folhas, diâmetro do coleto, massa seca da parte aérea e das raízes e índice de qualidade de Dickson. Efeitos significativos de promoção de crescimento foram observados com a aplicação isolada ou combinada de Trichoderma sp. e vermicomposto. A espécie $T$. asperelloides apresentou efeito superior a $T$. virens na promoção de crescimento de $J$. micrantha. Concluiu-se que o uso de vermicomposto e das espécies de Trichoderma sp. avaliadas promoveram o crescimento de $J$. micrantha, sendo que o uso combinado dos insumos biológicos promoveu incrementos mais pronunciados no crescimento.

\section{Growth promoters in propagation of caroba}

\begin{abstract}
The use of organic inputs, which are able of promoting plant growth, is an alternative to anticipate forest seedlings trading in nursery garden. Caroba (Jacaranda micrantha) is a native forest species from the southern of Brazil, which presents several potential uses. This study aims to analyze the efficiency of vermicompost and fungal isolates of the genus Trichoderma as growth promoters in J. micrantha seedlings. The experiment was carried out with nine substrates treatments, containing different percentages of vermicompost $(0,25$ and $50 \%)$ on the control substrate (sifted soil and commercial substrate Carolina Soil ${ }^{\circledR}$ in 1:1 ratio) in the presence or absence of $T$. asperelloides and $T$. virens. We used a completely randomized design with 18 repetitions per treatment. After 90 days of seeding we determined: shoot height, leaf number, stem diameter, dry weight of shoots and roots and Dickson quality index. Significant effects on growth promotion of caroba seedlings were observed for isolated or combined application of Trichoderma sp. and vermicompost. T. asperelloides isolate was superior then $T$. virens in promoting $J$. micrantha growth. It was concluded that the use of vermicompost and both species of Trichoderma sp. promoted growth of $J$. micrantha seedlings, and the combined use of both biologic inputs promoted more pronounced increase in plants growth.
\end{abstract}




\section{Introdução}

A falta de direcionamento técnico e de conscientização ecológica na exploração dos recursos florestais no Brasil tem acarretado prejuízos irreparáveis, ocasionando a extinção de espécies nativas de grande valor ecológico (Lorenzi, 2013).

Dentre as espécies florestais nativas da região sul do Brasil, Jacaranda micrantha Cham. (Bignoniaceae), popularmente conhecida como caroba, representa potencial para diferentes usos, sendo indicada tanto para recuperação de áreas degradadas, quanto para uso industrial e medicinal (Backes \& Irgang, 2002; Rovedder et al., 2016). É uma espécie pioneira encontrada, preferencialmente, em ecossistemas secundários. Devido à beleza de suas flores, é apreciada para a arborização e ornamentação de ruas e parques (Backes \& Irgang, 2002).

Na produção de mudas de qualidade, é indispensável a utilização de substratos que ofereçam características físicas e químicas adequadas, com boa estrutura, consistência, alta porosidade e capacidade de retenção de água e nutrientes (Urrestarazu et al., 2008; Belda et al., 2013). Dentre os materiais disponíveis para a composição de substratos visando à propagação de mudas, o vermicomposto, ou húmus de minhoca, tem se mostrado uma excelente alternativa, contribuindo para um adequado suprimento de nutrientes, bem como para a melhoria dos atributos físicos do substrato (Steffen et al., 2010). O vermicomposto é um material formado por substâncias orgânicas complexas e estáveis, resultante da atividade e interação de minhocas com microrganismos que habitam seu trato digestivo (Edwards et al., 2010). Apresenta influência direta e indireta sobre o crescimento das plantas, devido à presença de ácidos húmicos e hormônios reguladores do crescimento vegetal (Arancon et al., 2006; Ravindran et al., 2016; Maji et al., 2017).

Nos últimos anos, a utilização do vermicomposto para constituição de substratos tem apresentado resultados promissores na produção de mudas de hortaliças, espécies ornamentais e espécies florestais (Zaller, 2007; Steffen et al., 2010; Antunes et al., 2016). Aliada à prática de melhoria da qualidade dos substratos utilizados na produção de mudas florestais, a inoculação de microrganismos benéficos e promotores de crescimento vegetal representa uma alternativa eficiente e viável para uso em viveiros (Muthukumar \& Udaiyan, 2010).
Dentre os microrganismos mais utilizados na promoção do crescimento e proteção de plantas contra fitopatógenos, espécies do gênero Trichoderma têm se destacado. Esses fungos são encontrados naturalmente em quase todos os tipos de solo e possuem importante função ecológica, participando da decomposição e mineralização dos resíduos vegetais, o que favorece a disponibilização de nutrientes para as plantas (Esposito \& Silva, 1998; Zeilinger et al., 2016). Além disso, alguns isolados apresentam comprovada capacidade de produção de ácido indolacético (AIA) (Gravel et al., 2007), citocinina, giberelina e enzimas solubilizadoras de fosfato (Resende et al., 2014; Chagas et al., 2016). A estes metabólitos produzidos por alguns isolados de Trichoderma sp. têm sido atribuído efeito de estímulo ao enraizamento de plantas, bem como uma das estratégias dispensadas nas relações antagonistas (Gravel et al., 2007, Chagas et al., 2016). De acordo com Chen et al. (2011), o elevado potencial de algumas espécies de Trichoderma em disponibilizar nutrientes pode reduzir a necessidade de adubação em algumas culturas, configurando assim o seu potencial na promoção do crescimento vegetal. Embora diversos trabalhos demonstrem benefícios do uso de Trichoderma sp. sobre a produção de culturas agrícolas, a sua aplicação em espécies florestais nativas ainda é restrita (Donoso et al., 2008).

Não há dúvidas de que mudas florestais devem ser sadias e resistentes a estresses, possibilitando que sobrevivam às condições adversas do meio (Dent \& Wright, 2009). Já foi demonstrado que a inoculação de Trichoderma sp. e o uso de vermicomposto na composição de substratos interferem positivamente na qualidade e sanidade vegetal (Carvalho Filho et al., 2008; Lourenço \& Coelho, 2009). No entanto, até o momento, não foram encontrados resultados da avaliação do uso de Trichoderma spp. na produção de mudas de caroba, bem como estudos sobre a sua combinação com vermicomposto. Desta forma, o objetivo desse trabalho foi avaliar a interação da aplicação de vermicomposto e isolados fúngicos de Trichoderma spp. na promoção de crescimento em mudas de $J$. micrantha em condições de viveiro. 


\section{Material e métodos}

Foram avaliados nove tratamentos referentes à constituição de substratos contendo diferentes percentuais de vermicomposto no substrato padrão, na presença ou não de dois isolados de Trichoderma sp. (Tabela 1).

Tabela 1. Composição dos substratos utilizados para produção de mudas de Jacaranda micrantha em tubetes plásticos.

\begin{tabular}{ccccc}
\hline Tratamentos & $\begin{array}{c}\text { Substrato } \\
\text { padrão }\end{array}$ & Vermicomposto & $\begin{array}{c}\text { Trichoderma } \\
\text { asperelloides }\end{array}$ & T. virens \\
\hline 1 & $100 \%$ & $0 \%$ & - & - \\
2 & $75 \%$ & $25 \%$ & - & - \\
3 & $50 \%$ & $50 \%$ & - & - \\
4 & $100 \%$ & $0 \%$ & + & - \\
5 & $75 \%$ & $25 \%$ & + & - \\
6 & $50 \%$ & $50 \%$ & + & - \\
7 & $100 \%$ & $0 \%$ & - & + \\
8 & $75 \%$ & $25 \%$ & - & + \\
9 & $50 \%$ & $50 \%$ & - & + \\
\hline
\end{tabular}

$+=$ presença do isolado; - = ausência do isolado

O substrato padrão foi constituído por solo peneirado e substrato comercial Carolina Soil ${ }^{\circledR}$ na proporção 1:1 (v/v). O solo utilizado correspondeu ao horizonte A de um Argissolo Vermelho-Amarelo distrófico arênico (Sistema..., 1999). O vermicomposto foi produzido a partir de esterco curtido de bovinos e minhocas da espécie Eisenia andrei Bouché (1972). Após 100 dias de transformação, o vermicomposto foi separado das minhocas e peneirado em malha de $2 \mathrm{~mm}$.

Os isolados de Trichoderma sp. testados pertencem à coleção de fungos benéficos do Centro de Pesquisa em Florestas da Fundação Estadual de Pesquisa Agropecuária (Fepagro). $\mathrm{O}$ isolado T. asperelloides (T02) foi obtido a partir de uma amostra de solo rizosférico de povoamento florestal misto dentro da área experimental da Fepagro Florestas (Santa Maria, RS). $\mathrm{O}$ isolado de $T$. virens (T10) foi obtido da superfície de sementes de flamboyant (Delonix regia (Bojer ex Hook.) Raf.), cujo lote encontrava-se em avaliação de germinação no Laboratório de Análise de Sementes da Fepagro Florestas. A multiplicação dos isolados foi realizada de acordo com Steffen \& Maldaner (2017), por meio da inoculação de grãos de arroz colonizados pelas culturas puras dos microrganismos em substrato comercial Carolina Soil ${ }^{\circledR}$. Após o período de incubação, foram constituídos os nove tratamentos.

Foram utilizadas sementes de J. micrantha, coletadas no Município de Júlio de Castilhos, RS, e pertencentes ao lote no 58/10 do banco de sementes da Fepagro Florestas, com $81 \%$ de germinação, $94,12 \%$ de pureza e $6,57 \mathrm{~g}$ equivalente ao peso de mil sementes.

As unidades experimentais constaram de tubetes $\left(175 \mathrm{~cm}^{3}\right)$ dispostos em grades plásticas no interior de casa de vegetação, os quais foram preenchidos com os substratos referentes aos tratamentos. Foram semeadas duas sementes de caroba por unidade experimental. Após a germinação, realizou-se o raleio deixando-se apenas uma plântula por tubete. $\mathrm{O}$ delineamento experimental adotado foi o inteiramente casualizado, com nove tratamentos e 18 repetições, totalizando 162 unidades experimentais.

As características química do solo, do vermicomposto e do substrato comercial Carolina Soil ${ }^{\circledR}$ estão apresentadas na Tabela 2.

Tabela 2. Caracterização química dos materiais utilizados para composição dos substratos.

\begin{tabular}{cccc}
\hline $\begin{array}{c}\text { Atributos } \\
\text { químicos }\end{array}$ & Solo & Vermicomposto & Carolina Soil \\
\hline pH água 1:1 & 5,4 & 6,3 & 5,53 \\
MO (\%) & 1,3 & 29,70 & 35,0 \\
$\mathrm{C} \mathrm{( \% )}$ & 0,75 & 17,23 & 20,4 \\
$\mathrm{~N} \mathrm{( \% )}$ & 0,065 & 1,33 & 0,11 \\
$\mathrm{Ca}$ & $3,3 \mathrm{cmolc} \mathrm{dm}^{-3}$ & $6,54 \mathrm{~g} \mathrm{~kg}^{-1}$ & $0,14 \mathrm{~g} \mathrm{~kg}^{-1}$ \\
$\mathrm{Mg}$ & $0,9 \mathrm{cmolc} \mathrm{dm}^{-3}$ & $4,58 \mathrm{~g} \mathrm{~kg}^{-1}$ & $0,63 \mathrm{~g} \mathrm{~kg}^{-1}$ \\
$\mathrm{P}$ & $6,0 \mathrm{mg} \mathrm{dm}^{-3}$ & $2,41 \mathrm{~g} \mathrm{~kg}^{-1}$ & $6 \times 10^{-5} \mathrm{~g} \mathrm{~kg}^{-1}$ \\
$\mathrm{~K}$ & $48,0 \mathrm{mg} \mathrm{dm}^{-3}$ & $19,05 \mathrm{~g} \mathrm{~kg}^{-1}$ & $3 \times 10^{-4} \mathrm{~g} \mathrm{~kg}^{-1}$ \\
\hline
\end{tabular}

$\mathrm{Na}$ amostra de solo, foram determinados: valor de $\mathrm{pH}$ em água (1:1), teores de fósforo e potássio disponíveis, extraídos por Mehlich-1, teores de cálcio e magnésio trocáveis, extraídos por $\mathrm{KCl} 1,0 \mathrm{~mol} \mathrm{~L}^{-1}$, percentuais de matéria orgânica, carbono e nitrogênio, conforme metodologia de Tedesco et al. (1995). Os teores totais de carbono e nitrogênio do vermicomposto foram determinados em analisador elementar (modelo FlashEA 1112, Thermo Electron Corporation, Milão, Itália) no Laboratório de Biotransformações do Carbono e Nitrogênio da Universidade Federal de Santa Maria. O teor de matéria orgânica do vermicomposto foi 
calculado multiplicando-se o teor de carbono orgânico por 1,724 (fator de Van Bemmelen). Os teores de fósforo, potássio, cálcio e magnésio do vermicomposto foram determinados conforme Tedesco et al. (1995). Os atributos químicos do substrato comercial foram disponibilizados pelo fabricante (Tabela 2).

Aos 90 dias após a semeadura, foram avaliadas as variáveis de crescimento das mudas: altura da parte aérea, número de folhas, diâmetro do coleto, massa seca da parte aérea e das raízes. A altura das mudas foi determinada com auxílio de régua graduada $(\mathrm{mm})$, através da medida do nível do substrato até a inserção da última folha. O diâmetro de coleto foi determinado com auxílio de paquímetro digital, sendo a leitura realizada ao nível do substrato. Para a determinação da massa seca da parte aérea e das raízes, as mudas foram separadas em parte aérea e sistema radicular, permanecendo em estufa com circulação forçada de ar a $60^{\circ} \mathrm{C}$, até a obtenção de massa constante. Com base nos parâmetros biométricos das mudas, foi calculado o índice de qualidade de Dickson (Dickson et al., 1960).

Os dados obtidos foram submetidos ao teste de comparação de médias Tukey a 5\%, empregando-se o software SISVAR 5.6 (Ferreira, 2011).

\section{Resultados}

Foram observados efeitos significativos de promoção de crescimento das mudas de caroba com a aplicação isolada ou combinada de vermicomposto, Trichoderma asperelloides e T. virens (Tabelas 3).

Tabela 3. Altura, diâmetro do coleto e número de folhas de mudas de caroba (Jacaranda micrantha) aos 90 dias após a semeadura, cultivadas em diferentes substratos.

\begin{tabular}{|c|c|c|c|c|}
\hline Tratamentos & Sem Trichoderma & T. asperelloides & $T$. virens & CV $(\%)$ \\
\hline & \multicolumn{4}{|c|}{ Altura da parte aérea $(\mathrm{cm})$} \\
\hline $0 \%$ Vermicomposto & $3,90 \mathrm{~b} \mathrm{~B}$ & $5,13 \mathrm{ab} A$ & 4,16 b B & 13,32 \\
\hline $25 \%$ Vermicomposto & 4,76 a $\mathrm{A}$ & $4,58 \mathrm{~b} \mathrm{~A}$ & $4,61 \mathrm{~b} \mathrm{~A}$ & 14,11 \\
\hline $50 \%$ Vermicomposto & 4,87 a C & 5,50 a B & 6,90 a $\mathrm{A}$ & 2,35 \\
\hline \multirow[t]{2}{*}{ CV $(\%)$} & 13,64 & 9,25 & 17,68 & \\
\hline & \multicolumn{4}{|c|}{ Diâmetro do coleto $(\mathrm{mm})$} \\
\hline $0 \%$ Vermicomposto & $1,30 \mathrm{~b} \mathrm{~B}$ & $2,02 \mathrm{ab} \mathrm{A}$ & 1,62 a B & 16,61 \\
\hline $25 \%$ Vermicomposto & 1,71 a $\mathrm{A}$ & $1,77 \mathrm{~b} \mathrm{~A}$ & 1,71 a $\mathrm{A}$ & 12,41 \\
\hline $50 \%$ Vermicomposto & 1,58 a B & 2,16 a $\mathrm{A}$ & 2,04 a A & 17,90 \\
\hline \multirow[t]{2}{*}{ CV $(\%)$} & 10,85 & 12,68 & 22,15 & \\
\hline & \multicolumn{4}{|c|}{ Número de folhas } \\
\hline $0 \%$ Vermicomposto & $7,25 \mathrm{~b} \mathrm{~A}$ & 8,50 a $\mathrm{A}$ & 7,25 a $\mathrm{A}$ & 13,80 \\
\hline $25 \%$ Vermicomposto & $8,25 \mathrm{~b} \mathrm{~A}$ & 8,25 a $A$ & 7,87 a A & 7,54 \\
\hline $50 \%$ Vermicomposto & 9,75 a $\mathrm{A}$ & 8,25 a $A$ & 8,50 a $\mathrm{A}$ & 15,12 \\
\hline CV $(\%)$ & 11,59 & 11,56 & 12,89 & \\
\hline
\end{tabular}

Embora a fertilidade do solo utilizado para condução do ensaio estivesse em níveis satisfatórios quanto ao $\mathrm{pH} \mathrm{e}$ medianos quanto aos teores de fósforo e potássio (Tabela 1), conforme a Comissão de Química e Fertilidade do Solo (CQFS-RS/SC) (Manual..., 2004), o crescimento em altura das mudas de caroba foi aumentado pela suplementação do substrato com as duas concentrações de vermicomposto, o que se pode observar claramente nos tratamentos em ausência de Trichoderma sp. (T2 e T3) (Tabela 3).
Comparando-se apenas o efeito da inoculação dos isolados de Trichoderma sp. no substrato em ausência de vermicomposto (T4 e T7), observou-se que as mudas apresentaram maior crescimento em altura quando inoculadas com o isolado T. asperelloides. No entanto, quando houve adição de $25 \%$ de vermicomposto na composição do substrato, não foi observada diferença entre os tratamentos em relação à inoculação dos isolados de Trichoderma sp. Contudo, quando ocorreu a combinação da inoculação dos isolados de Trichoderma 
sp. e uso de $50 \%$ de vermicomposto, verificou-se um incremento em altura das mudas de caroba, sendo que $\mathrm{o}$ isolado $T$. virens apresentou maior efeito quando comparado ao $T$. asperelloides (Tabela 3 ).

Resposta semelhante foi verificada para o diâmetro do coleto das mudas, que aumentou significativamente quando o substrato foi constituído por $50 \%$ de vermicomposto e inoculado com isolados de Trichoderma sp., em relação ao tratamento controle sem vermicomposto e sem inoculação (Tabela 3).

Considerando-se o número de folhas por planta de caroba aos 90 dias após a semeadura, observou-se diferença significativa apenas em relação à adição da maior dose de vermicomposto ao substrato (50\%). A inoculação dos isolados de Trichoderma sp. não promoveu aumento deste parâmetro na presença ou ausência de vermicomposto (Tabela 3).

Os dois isolados de Trichoderma sp. avaliados apresentaram diferença quanto ao seu potencial na promoção do crescimento na ausência de vermicomposto, sendo que o isolado de T. asperelloides mostrou-se superior ao de $T$. virens nos parâmetros altura e diâmetro do caule (Tabela 3 ), massa seca das raízes e massa seca da parte aérea (Tabela 4).

Tabela 4. Massas secas de raízes e da parte aérea de mudas de caroba (Jacaranda micrantha) aos 90 dias após a semeadura, cultivadas em diferentes substratos.

\begin{tabular}{ccccc}
\hline Tratamentos & Sem Trichoderma & T. asperelloides & T. virens & CV (\%) \\
\hline 0\% Vermicomposto & 34,80 a B & 108,70 a A & 61,13 a B & 21,15 \\
25\% Vermicomposto & 51,47 a B & 76,95 a A & 78,93 a A & 26,24 \\
$50 \%$ Vermicomposto & 39,52 a B & 121,81 a A & 55,25 a A & 24,38 \\
CV (\%) & 30,56 & 34,35 & 55,25 & \\
\hline \multicolumn{5}{c}{ Massa seca da parte aérea (mg) } \\
\hline 25\% Vermicomposto & 48,23 b C & 178,07 a A & 120,41 b B & 27,17 \\
$50 \%$ Vermicomposto & 100,30 a B & 148,16 a A & 134,18 ab A & 25,73 \\
CV (\%) & 19,75 a B & 206,18 a A & 191,60 a A & 27,16 \\
\hline
\end{tabular}

*Médias seguidas de mesma letra minúscula nas colunas e maiúscula nas linhas não diferem entre si pelo teste de Tukey $(\mathrm{P} \leq 0,05)$.

Ganhos significativos em biomassa das mudas foram obtidos com a inoculação dos fungos no substrato. Os valores de massa seca das raízes foram maiores com a adição de Trichoderma sp. ao substrato, com destaque para o isolado T. asperelloides. A adição de vermicomposto não influenciou a produção de massa seca de raízes. Por outro lado, o vermicomposto foi importante para a produção de biomassa da parte aérea das mudas na ausência de Trichoderma sp. A associação de vermicomposto com ambas as espécies de Trichoderma sp. promoveu ganhos significativos neste parâmetro (Tabela 4).

Valor mais elevado do índice de qualidade de Dickson (IQD) foi obtido no tratamento onde houve a adição de $50 \%$ de vermicomposto e inoculação do isolado de $T$. asperelloides (Tabela 5).
Tabela 5. Índice de qualidade de Dickson (IQD) de mudas de caroba (Jacaranda micrantha) aos 90 dias após a semeadura, cultivadas em diferentes substratos.

\begin{tabular}{cccc}
\hline Tratamentos & Sem Trichoderma & T. asperelloides & T. virens \\
\hline 0\% Vermicomposto & 0,019 & 0,068 & 0,039 \\
25\% Vermicomposto & 0,031 & 0,049 & 0,047 \\
$50 \%$ Vermicomposto & 0,024 & 0,079 & 0,060 \\
\hline
\end{tabular}

\section{Discussão}

A variável diâmetro do coleto é o parâmetro mais utilizado para indicar a capacidade de sobrevivência das mudas em campo, sendo por isso o mais recomendado para definição das doses de fertilizantes a serem aplicadas na produção de mudas (Daniel et al., 1997). Segundo Souza et al. (2006), existe uma grande

Pesq. flor. bras., Colombo, v. 37, n. 90, p. 149-157, abr./jun. 2017 
correlação entre a percentagem de sobrevivência de mudas após o plantio em campo e o diâmetro do coleto. No presente estudo, plantas de caroba crescidas em substrato com a inoculação de Trichoderma sp. e a adição de vermicomposto ao substrato apresentaram maiores médias de diâmetro do coleto em relação ao tratamento controle (Tabela 3).

Mesmo ainda não sendo totalmente compreendidos os mecanismos bioquímicos da ação do vermicomposto sobre o crescimento vegetal, muitos indícios apontam características que permitem compará-los ao efeito da adição de auxinas, giberelinas e citocininas (Rodda et al., 2006; Masciandaro et al., 2014), fitorreguladores que atuam no crescimento e desenvolvimento dos vegetais, agindo como transportadores de açúcares na parede celular e na síntese de glicoproteínas (Taiz \& Zeiger, 2013).

O potencial do vermicomposto na promoção do crescimento de mudas de espécies florestais já foi reportado em outros trabalhos (Caldeira et al., 2008; Steffen et al., 2011; Andreazza et al., 2013; Antunes et al., 2016). Esse efeito é atribuído ao teor de nutrientes (Tabela 2), bem como às quantidades significativas de ácidos húmicos e hormônios reguladores do crescimento vegetal que apresenta o substrato quando constituído por vermicomposto (Arancon et al., 2006; Ravindran et al., 2016). Além disso, o vermicomposto proporciona um crescimento favorável da população microbiana que, por sua vez, participa da biodisponibilização de nutrientes minerais para as plantas, influenciando assim o crescimento vegetal (Ravindran et al., 2016; Maji et al., 2017). Possivelmente, o efeito benéfico do vermicomposto sobre o crescimento dos microrganismos explique o maior efeito de promoção de crescimento nas mudas de caroba quando foi realizada a inoculação dos isolados de Trichoderma sp. em substrato constituído por $50 \%$ de vermicomposto (Tabela 3 ).

De acordo com Shoresh et al. (2010), alguns isolados de Trichoderma sp. promovem efeitos diretos sobre as plantas, aumentando seu potencial de crescimento e absorção de nutrientes, a eficiência no uso de fertilizantes, o percentual de germinação das sementes, bem como o estímulo às defesas das plantas contra estresses bióticos e abióticos. Diferentes culturas agrícolas já tiveram seu crescimento estimulado por Trichoderma sp., especialmente na produção de biomassa, como soja, milho, feijão (Tsahouridou \& Thanassoulopoulos, 2002) e maracujazeiro (Santos et al., 2010).
Os maiores valores médios de massa seca da parte aérea foram observados no substrato com a inoculação de Trichoderma sp. (Tabela 4). Dentre os fatores envolvidos na promoção do crescimento das mudas pelos isolados de Trichoderma sp., a produção de determinados metabólitos por esses fungos pode estimular a multiplicação celular vegetal, conforme reportado por Aguiar et al. (2015). Os autores observaram que diferentes isolados de Trichoderma sp. apresentam diferenças na capacidade de aumentar o índice mitótico de células do ápice de raízes de Allium cepa $\mathrm{L}$. De acordo com Carvajal et al. (2009), alguns destes metabólitos têm atividades reconhecidamente análogas aos reguladores de crescimento vegetal.

Diferenças entre os isolados são esperadas, uma vez que é sabido que enquanto algumas linhagens aumentam a superfície total do sistema radicular, possibilitando um maior acesso aos elementos minerais (Harman, 2004), outras são mais eficientes em solubilizar e disponibilizar para a planta, nutrientes presentes no solo. Há ainda aqueles isolados que otimizam os mecanismos ativos de absorção, aumentando a eficiência da planta para utilizar alguns nutrientes importantes, como o fósforo e o nitrogênio (Altamore et al., 1999; Zeilinger et al., 2016). Assim, essa diferença pode refletir mais as necessidades determinadas pela planta em estudo do que o próprio potencial do isolado de Trichoderma sp. Diferenças entre os isolados de T. asperelloides e T. virens na promoção de crescimento foram observadas no presente estudo, sendo que a presença de vermicomposto no substrato interferiu na eficiência dos isolados (Tabelas 3 e 4).

Para a caroba, o trabalho realizado por Tedesco et al. (1999) apresenta-se como a única publicação referente à utilização de doses de vermicomposto na composição do substrato para produção de mudas. Os referidos autores avaliaram o efeito de doses crescentes de vermicomposto $(0,20,40,60$ e $80 \%$ do volume do substrato) sobre o crescimento e a qualidade de mudas ao longo de 120 dias. Os resultados demonstraram relação direta entre o aumento da dosagem de vermicomposto no substrato e os parâmetros altura das mudas e biomassa (aérea e radicular), sendo indicado o uso entre 60 e $80 \%$ do volume do tubete. Os resultados observados no presente trabalho corroboram com os apresentados por Tedesco et al. (1999), demonstrando que doses mais elevadas de vermicomposto proporcionam maior incremento no crescimento de mudas de caroba (Tabelas 3 e 4). 
O índice de qualidade de Dickson (IQD) reflete bem o efeito dos tratamentos no crescimento da mudas de caroba, sendo que os fatores vermicomposto e Trichoderma spp. promoveram, isoladamente, o crescimento vegetal, que foi ainda mais incrementado com a combinação dos dois fatores. $\mathrm{O}$ maior valor do IQD, que indica melhor qualidade de mudas, foi obtido no tratamento onde houve a adição de $50 \%$ de vermicomposto e inoculação do isolado T. asperelloides. Essa resposta foi ainda mais evidente pelo fato de que não foi atingido o valor mínimo padrão para o IQD das mudas, que é de 0,20 conforme recomendação de Hunt (1990), no tratamento em que tanto Trichoderma sp. quanto vermicomposto estiveram ausentes (Tabela 5).

Segundo Azevedo et al. (2010), o IQD é utilizado por considerar o vigor e o equilíbrio da distribuição da biomassa na muda. No presente estudo, com a utilização de T. asperelloides e T. virens em substrato contendo 50\% de vermicomposto, foi possível aumentar a qualidade (IQD) da mudas em 315,7 e 215,7\% respectivamente, em relação ao tratamento controle (Tabela 5).

\section{Conclusões}

O uso combinado dos insumos biológicos vermicomposto e isolados fúngicos do gênero Trichoderma representa uma ferramenta eficiente para a produção de mudas de Jacaranda micrantha em ambiente de viveiro, promovendo o crescimento vegetal e antecipando o momento de comercialização das mudas. $\mathrm{Na}$ ausência de vermicomposto no substrato, o isolado de T. asperelloides foi superior ao de T. virens na promoção de crescimento, elevando os valores de altura, diâmetro do coleto, índice de qualidade de Dickson, massa seca da parte aérea e das raízes. No entanto, em substrato contendo $50 \%$ de vermicomposto, $T$. asperelloides e $T$. virens não diferiram estatisticamente quanto à produção de massa seca da parte aérea, elevando em 327,49 e $297,26 \%$, respectivamente, os valores deste parâmetro em relação ao tratamento onde não foram utilizados os insumos biológicos.

\section{Agradecimentos}

À Fundação de Amparo à Pesquisa do Estado do Rio Grande do Sul (FAPERGS) pelo apoio financeiro.

\section{Referências}

Aguiar, A. R. et al. Efeito de metabólitos produzidos por Trichodemrma spp. sobre o índice mitótico em células das pontas de raízes de Allium cepa. Bioscience Journal, v. 31, n. 3, p. 934-940, 2015. DOI: 10.14393/BJ-v31n3a2015-23292.

Altamore, C. et al. Solubilization of phosphates and micronutrients by the plant-growth-promoting and biocontrol fungus Trichoderma harzianum Rifai 1295-22. Applied and Environmental Microbiology, v. 65, n. 7, p. 2926-2933, 1999. DOI: 10.1371/ journal.pone.0130081.

Andreazza, R. et al. Efeito do vermicomposto no crescimento inicial de ipê amarelo (Handroanthus chrysotrichus) e leucena (Leucaena leucocephala). Nativa, v. 1, n. 1, p. 29-33, 2013. DOI: 10.14583/2318-7670.v01n01a06.

Antunes, R. M. et al. Crescimento inicial de acácia-negra com vermicompostos de diferentes resíduos agroindustriais. Ciência Florestal, v. 26, n. 1, p. 1-9, 2016. DOI: 10.5902/1980509821060.

Arancon, N. Q. et al. Effects of humic acids from vermicomposts on plant growth. European Journal of Soil Biology, v. 42, n. 1, p. 65-69, 2006. DOI: 10.1016/j.ejsobi.2006.06.004.

Azevedo, I. M. G. et al. O. Estudo do crescimento e qualidade de mudas de marupá (Simarouba amara Aubl) em viveiro. Acta Amazônica, v. 40, n. 1, p. 157-164, 2010. DOI: 10.1590/S004459672010000100020 .

Backes, P. \& Irgang, B. Árvores do Sul: guia de identificação e interesse ecológico: as principais espécies nativas sul-brasileiras. Santa Cruz do Sul: Instituto Souza Cruz, 2002.

Belda, R. M. et al. Nutrient-rich compost versus nutrient-poor vermicompost as growth media for ornamental-plant production. Journal Plant Nutrition Soil Science, v. 176, n. 6, p. 827-835, 2013. DOI: 10.1002/jpln.201200325.

Caldeira, M. V. W. et al. Composto orgânico na produção de mudas de aroeira vermelha. Scientia Agrária, v. 9, n. 1, p. 27-33, 2008. DOI: $10.5380 /$ rsa.v9i1.9898.

Carvajal, L. H. et al. Growth stimulation in bean (Phaseolus vulgaris L.) by Trichoderma. Biological Control, v. 51, p. 409-416, 2009. DOI: 10.1016/j.biocontrol.2009.07.018.

Carvalho Filho, M. R. et al. Avaliação de isolados de Trichoderma na promoção de crescimento, produção de acido indolacético in vitro e colonização endofítica de mudas de eucalipto. Brasília, DF: Embrapa Recursos Genéticos e Biotecnologia, 2008. 16 p. (Embrapa Recursos Genéticos e Biotecnologia. Documentos, 226).

Chagas, L. F. B. et al. Efficiency of Trichoderma spp. as a growth promoter of cowpea (Vigna unguiculata) and analysis of phosphate solubilization and indole acetic acid synthesis. Brazillian Journal of Botany, v. 39, n. 2, p. 437-445, 2016. DOI: 10.1007/s40415015-0247-6.

Chen, L. H. et al. Trichoderma harzianum SQR-T037 rapidly degrades allelochemicals in rhizospheres of continuously cropped cucumbers. Applied Microbiology and Biotechnology, v. 89, p. 1653-1663, 2011. DOI: 10.1007/s00253-010-2948-x. 
Daniel, O. et al. Aplicação de fósforo em mudas de Acacia mangium WILLD. Revista Árvore, v. 21, n. 2, p. 163-168, 1997.

Dent, D. H. \& Wright, S. J. The future of tropical species in secondary forest: a quantitative review. Biological Conservation, v. 142, p. 2833-2843, 2009. DOI: 10.1016/j.biocon.2009.05.035.

Dickson, A. et al. Quality appraisal of white spruce and white pine seedling stock in nurseries. Forestry Chronicle, v. 36, p. 10-13, 1960. DOI: $10.5558 / \mathrm{tfc} 36010-1$.

Donoso, E. et al. Efecto de Trichoderma harzianum y compost sobre el crecimiento de plántulas de Pinus radiata em viveiro. Bosque, v. 29, n. 1, p. 52-57, 2008. DOI: 10.4067/S0717-92002008000100006.

Edwards, C. A. et al. Vermiculture technology: earthworms, organic wastes, and environmental management. New York: CRC Press, 2010. DOI: $10.1201 / \mathrm{b} 10453$.

Esposito, E. \& Silva, M. Systematics and environmental application of the genus Trichoderma. Critical Reviews in Microbiology, v. 24, n. 2, p. 89-98, 1998. DOI: 10.1080/10408419891294190.

Ferreira, D. F. Sisvar: a computer statistical analysis system. Ciência e Agrotecnologia, v. 35, n. 6, p. 1039-1042, 2011. DOI: 10.1590/ S1413-70542011000600001.

Gravel, V. et al. Growth stimulation and fruit yield improvement of greenhouse tomato plants by inoculation with Pseudomonas putida or Trichoderma atroviride possible role of indole acetic acid (IAA). Soil Biology \& Biochemistry, v. 39, p. 1968-1977, 2007. DOI: 10.1016/j.soilbio.2007.02.015.

Harman, G. E. et al. Trichoderma species: opportunistic, avirulent plant symbionts. Nature Reviews Microbiology, v. 2, n. 1, p. 43-56, 2004. DOI: $10.1038 /$ nrmicro797.

Hunt, G. A. Effect of styroblock design and cooper treatment on morphology of conifer seedlings. In: Rose, R. et al. (Ed.). Proceedings... Fort Collins: U.S. Department of Agriculture, Forest Service, Rocky Mountain Forest and Range Experiment Station, 1990. p. 218-222. (Roseburg, OR. General Technical Report RM200).

Lorenzi, H. Árvores brasileiras: manual de identificação e cultivo de plantas arbóreas nativas do Brasil. 4. ed. Nova Odessa: Plantarum, 2013. v. 2. 384 p.

Lourenço, N. M. G. \& Coelho, S. I. D. Vermicompostagem e qualidade ambiental. São Bartolomeu de Messines, 2009.

Maji, D. et al. Humic acid rich vermicompost promotes plant growth by improving microbial community structure of soil as well as root nodulation and mycorrhizal colonization in the roots of Pisum sativum. Applied Soil Ecology, v. 110, p. 97-108, 2017. DOI: 10.1016/j.apsoil.2016.10.008.

Manual de adubação e de calagem para os estados do Rio Grande do Sul e de Santa Catarina. SBCS/NRS. Porto Alegre: Sociedade Brasileira de Ciência do Solo, Núcleo Regional Sul, Comissão de Química e Fertilidade do Solo, 2004. 400 p.

Masciandaro, G. et al. Fertigation with wastewater and vermicompost: soil biochemical and agronomic implications. Pedosphere, v. 24, n. 5, p. 625-634, 2014. DOI: 10.1016/S1002-0160(14)60048-5.
Muthukumar, T. \& Udaiyan, K. Growth response and nutrient utilization of Casuarina equisetifolia seedlings inoculated with bioinoculants under tropical nursery conditions. New Forests, v. 40, n. 1, p. 101-118, 2010. DOI: 10.1007/s11056-009-9186-Z.

Ravindran, B. et al. Influence of microbial diversity and plant growth hormones in compost and vermicompost from fermented tannery waste. Bioresource Technology, v. 217, p. 200-204, 2016. DOI: 10.1016/j.biortech.2016.03.032.

Resende, M. P. et al. Phosphate solubilization and phytohormone production by endophytic and rhizosphere Trichoderma isolates of guanandi (Calophyllum brasiliense Cambess). African Journal of Microbiology Research, v. 8, n. 27, p. 2616-2623, 2014. DOI: 10.1007/s40415-015-0247-6.

Rodda, M. R. C. et al. Estímulo no crescimento e na hidrólise de ATP em raízes de alface tratadas com humatos de vermicomposto. I- Efeito da concentração. Revista Brasileira de Ciência do Solo, v. 30, n. 4, p. 649-656, 2006. DOI: 10.1590/S0100-06832006000400005.

Rovedder, A. P. M. et al. Potential medicinal use of forest species of the Deciduous Seasonal Forest from Atlantic Forest Biome, South Brazil. Brazilian Archives of Biology and Technology, v. 59, p. 1-11, 2016. DOI: 10.1590/1678-4324-2016150329.

Santos, H. A. et al. Associação de isolados de Trichoderma ssp. E ácido indol - 3- butírico (AIB) na promoção de enraizamento de estacas e crescimento de maracujazeiro. Bioscience Journal, v. 26, n. 6, p. 966-972, 2010.

Shoresh, M. et al. Induced systemic resistance and plant responses to fungal biocontrol agents. Annual Review of Phytopathology, v. 48, p. 21-43, 2010. DOI: 10.1146/annurev-phyto-073009-114450.

Sistema Brasileiro de Classificação de Solos. Brasília, DF: Embrapa Produção de Informação; Rio de Janeiro: Embrapa Solos, 1999.

Souza, C. A. M. et al. Crescimento em campo de espécies florestais em diferentes condições de adubação. Ciência Florestal, v. 16, n. 3, p. 243-249, 2006. DOI: 10.5902/198050981905.

Steffen, G. P. K. et al. Húmus de esterco bovino e casca de arroz carbonizada como substratos para a produção de mudas de boca-deleão. Acta Zoológica Mexicana, v. 26, nesp. 2, p. 345-357, 2010.

Steffen, G. P. K. et al. Utilização de vermicomposto como substrato na produção de mudas de Eucalyptus grandis e Corymbia citriodora. Pesquisa Florestal Brasileira, v. 3, n. 36, p. 75-82, 2011. DOI: 10.4336/2011.pfb.31.66.75.

Steffen, G. P. K. \& Maldaner, J. Methodology for Trichoderma sp. multiplication in organic substrates. International Journal of Current Research, v. 9, n. 1, p. 44564-44567, 2017.

Taiz, L. \& Zeiger, E. Fisiologia vegetal. 5. ed. Porto Alegre: ArtMed, 2013. 954 p.

Tedesco, J. et al. Análise de solo, planta e outros materiais. 2. ed. Porto Alegre: UFRGS, 1995. 174 p.

Tedesco, N. et al. Influência do vermicomposto na produção de mudas de caroba (Jacaranda micrantha Chamisso). Revista Árvore, v. 23, n. 1, p.1-8, 1999. 
Tsahouridou, P. C. \& Thanassoulopoulos, C. C. Proliferation of Trichoderma koningii in the tomato rhizosphere and the suppression of damping-off by Sclerotium rolfsii. Soil Biology \& Biochemistry, v. 34, n. 6, p. 767-776, 2002. DOI: 10.1016/S0038-0717(02)00006-8.

Urrestarazu, M. et al. Wetting agent effect on physical properties of new and reused rockwool and coconut coir waste. Scientia Horticulturae, v. 116, n. 1, p. 104-108, 2008. DOI: 10.1016/j. scienta.2007.10.030.
Zaller, J. G. Vermicompost as a substitute for peat in potting media: effects on germination, biomass allocation, yields and fruit quality of three tomato varieties. Scientia Horticulturae, v. 112, n. 2, p. 191-199, 2007. DOI: 10.1016/j.scienta.2006.12.023.

Zeilinger, S. et al. Secondary metabolism in Trichoderma: chemistry meets genomics. Fungal Biology Reviews, v. 30, n. 2, p. 74-90, 2016. DOI: 10.1016/j.fbr.2016.05.001. 
